\title{
Principles of protein structure: an established Internet-based course in structural biology
}

\author{
Clare Sansom and David Moss \\ Department of Crystallography, Birkbeck College \\ email:d.moss@mail.crystbbk.ac.uk
}

The Department of Crystallography at Birkbeck College, London, UK, has been running a one-year, part-time accredited graduate course, 'Principles of Protein Structure', entirely over the Internet since 1996. Students on this course learn the basic principles of the increasingly important subject of structural biology using software programs such as Rasmol and Chime to visualize and manipulate molecular structures in three dimensions. They interact with their tutors, based at Birkbeck, using email and text-based teleconferencing, and can test their knowledge with multiple choice quizzes on the Web. Over 200 students from thirty countries registered for this course in the last four years. Forty, from central and eastern Europe, were supported by bursaries from the Open Society Institute. The course has been well received by students and its success led us to introduce a similar course in protein crystallography.

\section{Introduction}

The Internet is becoming an important medium for the delivery of educational materials. However, relatively few institutions are delivering whole courses using this medium. More often, the technologies are used to complement traditional courses, which may be given face-to-face or at a distance (Farrell, 1999). The Department of Crystallography at Birkbeck College, London, has been in the vanguard of the development of 'virtual education', providing some of the first accredited postgraduate courses in the UK to be offered entirely using the new technologies. For the past four years, we have been running an Advanced Certificate course entitled 'Principles of Protein Structure using the Internet'l (Sansom, Walshaw and Moss, 1997) (PPS). See http://www.cryst.bbk.ac.uklpps for more details. This was one of the first tutor-assisted, accredited, university-level courses to be taught entirely over the Internet, and is certainly the first in biochemistry in the UK. 
In 1997 the first delivery of the accredited course was granted an award from the Universities and Colleges Information Systems Association (UCISA) for innovative use of the Web in higher education. The PPS course is of nine months' duration, and of final-year undergraduate standard, although almost all students are graduates. Successful students receive the award of an Advanced Certificate from London University, which is a graduate level qualification approximately equivalent to half a UK M.Sc. degree. The success of the PPS course has encouraged the department to introduce a second Advanced Certificate, in Protein Crystallography, taught in this way.

Our students, who may come from any country, can study at home, at work or at college, and set their own hours of study. This is self-evidently also true for more conventional types of distance education, delivered through paper-based and broadcast media. However, this way of providing education differs from traditional types of distance learning in that both delivery of course material and student-tutor interactions take place using only Internet and associated multimedia technologies. Students are expected to have regular access to a computer with a reliable Internet connection and access to the Web via a modern graphical browser such as Netscape or Internet Explorer version 3 or 4. Currently, we support students using PC, Macintosh and Unix platforms. The minimum acceptable specification is kept fairly low; a 486 or an equivalent machine of another platform is sufficient. This technology is readily and fairly cheaply available almost everywhere in the world. Most of our students use PCs, or Unix workstations from Sun and Silicon Graphics, and these are the best supported by the technical staff at Birkbeck. We deliberately chose to keep the minimum specification low and to allow students choice of several platforms, although this has limited our choice of software. The benefit of this, of course, is in increasing access to the course for a wide range of students.

Although all the course tutors are based at Birkbeck, the students also benefit from the involvement of a group of consultants. These people are usually academics from the UK or abroad who conduct tutorials on specialized topics in the syllabus and are available to answer students' questions by email. Some consultants have also assisted in the electronic administration of the courses.

The developments that led to the PPS course started in 1993, with the production of software for the display of macromolecular structure for teaching crystallography at Birkbeck. The growing popularity of the Web led to the next step: the idea of delivering this software over the Web for students' use away from Birkbeck. A pilot version of the PPS course was launched early in 1995 to evaluate the possibilities of these technologies for the delivery of a full-scale course. The success of this led to the launch of the accredited course, in 1996. A total of 252 students have registered for the accredited course in the five years that it has been running. Our students have come from thirty countries, covering every continent except Africa. Just over 30 per cent (seventy-nine) are women; nine were over fifty-five when they started the course. The drop-out rate for the accredited courses has been low. In order to be accepted for an Advanced Certificate, students need a UK bachelor's degree in any science subject or its equivalent. A few undergraduate students have been accepted on the courses. These are usually from central European countries, where some undergraduate programmes are up to five years long and lead to the award of the equivalent of a master's degree. 


\section{Syllabus and assessment}

The choice of bioinformatics and protein structure, including crystallography, as the core scientific subjects taught reflects the growing importance of these subjects to industry. Structures of protein molecules are now being determined at an accelerating rate, driven by technological improvements and increased investment in molecular biology through genome projects. The design of novel drugs and vaccines depends on an understanding of the details of the relationships between protein sequence, structure and function. The pharmaceutical and biotechnology industries increasingly need personnel trained to a high standard in these interdisciplinary subjects. What was, only ten years ago, an esoteric subdiscipline is becoming a necessary part of modern biology. Bioinformatics and structural biology are becoming 'core skills' for graduate biologists, and the pace of technological change is such that lifelong learning must become part of the culture. The syllabus of the PPS course addresses the skill shortage in this area by introducing graduate students, and updating inexperienced scientists, in the knowledge and skills needed to take part in structural molecular biology research. It starts with an introduction to the use of Internet tools and protocols, followed by basic protein structure, protein synthesis, elementary bioinformatics (DNA and protein sequence analysis) and the relationship between protein structure and function.

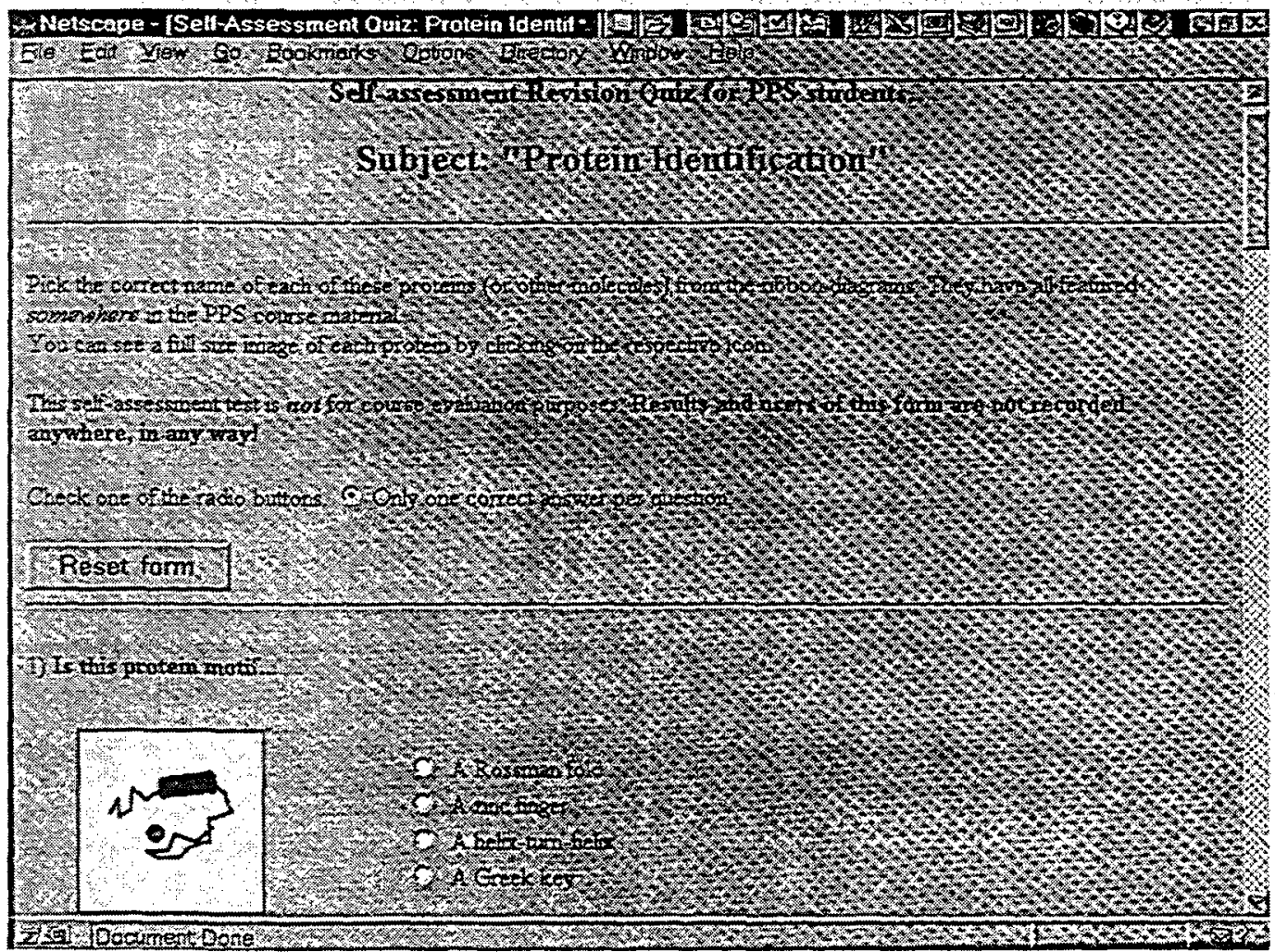

Figure 1: Screen-shot illustrating a Protein Identificotion Quiz used for self-assessment in the 1998-9 version of the Principles of Protein Structure course. 
Formal assessment of the PPS course consists of an examination and a project. The examination is the only part of the course that is not Internet-based. This is conducted along the same lines as the UK's Open University examinations but on a much smaller scale. Students attend in person at one of a number of local centres temporarily established throughout the world. This is an important means of verifying the students' identities. The project consists of a simple resource written in HTML to present and explain some aspect of protein structure. We emphasize that the students are not expected to become experts in Web design and programming, just to learn enough HTML to present an attractive and well-linked electronic review. Some example projects are mounted on the Web to give future students an idea of what is expected.

Students also benefit from exercises set throughout the course, which do not contribute to their assessment. Tutors may use the interactive and multimedia functionality of the Web to set course-work exercises containing, for example, images of protein structures which the students are expected to manipulate. Answers to questions may be submitted via email or hypertext forms. This electronic submission of answers permits automatic checks to be made on student progress. Each student on the protein crystallography course is assigned a separate user identifier, which they use to run calculations on the central computer facilities at Birkbeck, both for self-assessment and for their project work. We have also provided multiple-choice quizzes with instant feedback as self-assessment exercises (Figure 1).

\section{International implications}

Access to PPS and the other Internet-based Advanced Certificate courses is extremely valuable in countries where the techniques of bioinformatics and structural biology are relatively poorly developed. Many countries in central Europe, which are currently preparing for entry to the European Union, are particularly well placed to benefit from these courses. Although the first protein crystallography laboratories opened in Poland and Hungary in 1994, these countries still suffer badly from skill shortages in these subjects. A crystallography group was formed in Prague (Czech Republic) in 1998. Although the economies of these countries are growing, disciplines such as crystallography have not yet developed to a point where students can readily access training programs locally.

One advantage of distance education is that students from emerging economic areas can take part in western higher education programmes at a fraction of the cost of travelling to, and living in, western countries. The level of technology required to benefit from the Birkbeck courses is increasingly available throughout less developed regions, particularly central Europe and the former Soviet Union, South America and parts of east Asia. However, the fees introduced for the accredited course, although much lower than those for similar, traditional courses, effectively denied access to these students. Birkbeck College was awarded a two-year grant from the Open Society Institute that enabled it to offer bursaries to about twenty students from central Europe and the former Soviet Union in 1997 and again in 1998. Although the fees for students located outside the 'western world' have now been reduced to the level paid by UK- or EU-based students, the loss of the bursaries has led to a serious decline in student numbers in the 1998-9 academic year. Three students from Poland and one from the Czech Republic are currently taking the courses. We are currently exploring other possibilities for supporting students from these regions. 


\section{Enabling technologies}

The use of the Web as a tool to deliver educational materials and email discussion lists for asynchronous communication between tutors and students are well-established techniques in distance learning. A large proportion of the course material has been developed 'inhouse' (see Figure 2 for an example). Much 'legacy material', prepared for conventional teaching at Birkbeck, has been rewritten for the Web (King, 1998). This process, although straightforward, is fairly time-consuming. We also provide access to high-quality tutorial or reference material relevant to the syllabus available elsewhere on the Internet. We have eliminated potential copyright problems by referencing the material using hypertext links, with the permission of the authors. Messages on the lists are placed in an archive catalogued according to date and topic. Students can review these archives and follow discussions that have taken place earlier in the course. The unique mixture of technologies used in this course also includes molecular visualization software and a text-based networked virtual environment (TNVE) as a synchronous communication tool for tutorials.

The study of three-dimensional protein structure is made easier by being able to rotate colour images of the molecules on the computer screen. In PPS we provide viewing software such as Rasmol (Sayle, 1999) and associated scripts that enable students to rotate

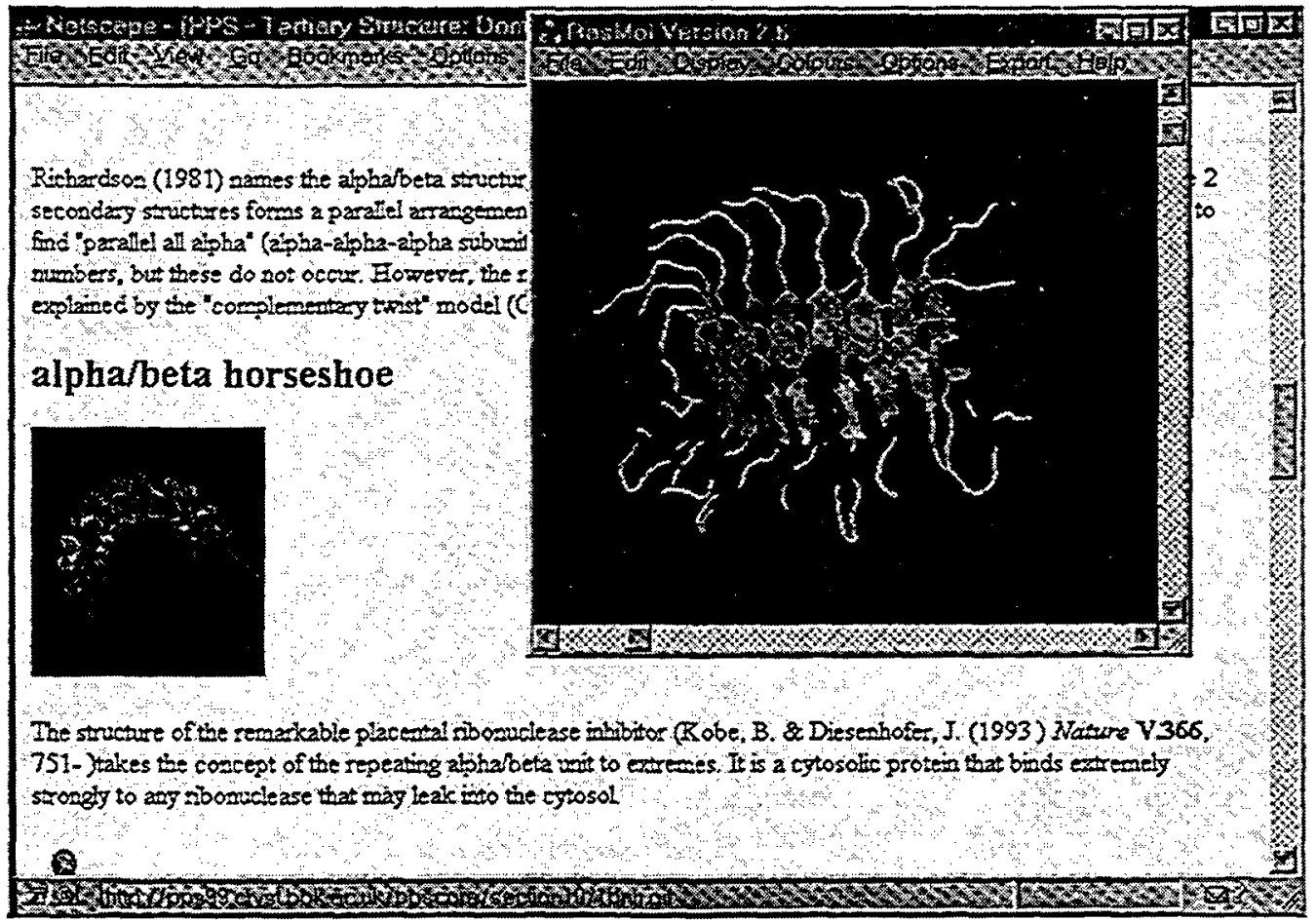

Figure 2: Screen-shot of a section of course material illustrating human ribonudease inhibitor (PDB code ( $04 y$ ), a protein which has an unusual 'alpholbeta horseshoe' structure. The inset shows the same protein displayed using the molecular viewer Rosmol. 
ClareS says 'in this meeting (and to-morrow's late night session) we will be discussing section 4 of the course material: on protein synthesis'

ClareS says 'a phrase that is used to cover the process of transcription / translation, protein maturation and targeting, and even protein degradation'

ClareS says 'does anyone want to start with a question?' ClareS looks round expectantly ...

KLaurio says 'the codons can code for 64 different AAs, there are 20 common AAs in proteins'

ClareS says 'yes - that is redundancy'

KLaurio says 'does all the tRNA really use all the 3 bases in the codon for targeting?'

Geraint says 'why (or when) did eukaryotes give up on coordinated transcription?'

KLaurio says 'perhaps the most common proteins only use 2'

ClareS says 'if you look at the code you will see that sometimes all 3 bases are used to distinguish amino acids'

ClareS says 'if only 2 positions were regularly used there could only reliably be 16 amino acids'

Geraint says 'are the limited number of genes in mitochondrial or chloroplast DNA transcribed coordinately!"

ClareS says 'on the other hand, the 'wobble base' is almost always the 3rd - it may well be that interactions between this base and the tRNA are weaker'

KLaurio says 'ok, l'll look into the books :)'

ClareS [to Geraint] good questions - only problem is that I don't know the answers... David, can you help...?

Clares [to KLaurio] there are a few structures of tRNA / codon complexes in PDB - if I've remembered it correctly

KLaurio says in the material they suggested we disable the water to get a better view of the DNA molecule...'

Figure 3: Example of dialogue from a PPS tutorial held in BioMOO on 6 january 1999. The students are discussing protein synthesis with Dr Clare Sansom (course tutor).

protein images on their computers. Rasmol is readily accessible over the Internet, can be easily installed on a wide variety of computer platforms and, importantly, it is free. We are gradually increasing our use of the Web plug-in, Chime, which is partly based on Rasmol. Chime is an important teaching resource as it is relatively straightforward to combine with Javascript to produce interactive tutorials (MDLI, 1999). Unfortunately, as later versions are only available for the Windows and Mac operating systems, we will not be able to replace Rasmol with Chime if we wish to carry on supporting students using Unix workstations. Molecular movies are very useful for helping students gain an understanding of complex molecular processes such as muscle contraction. Students also use the program Mage to display interactive diagrams or 'kinemages' illustrating protein structure, 
including those produced for use with the excellent textbook Introduction to Protein Structure (Branden and Tooze, 1999).

Interactive tutorials, which are run using a MUD (a Multi-User Dimension) or a MOO (an object-oriented MUD) are a popular feature of the course. When a tutor (usually based at Birkbeck) and a group of students (who may be scattered throughout the world) are connected simultaneously to such a system, the tutor can write text which the students can see almost instantaneously and reply in real time. For the first four years of the course, we used 'BioMOO' (Sansom, 1997; Towell and Towell, 1997), which is mounted at the Weizmann Institute in Israel. Figure 3 shows a dialogue taken from a real tutorial held in BioMOO in the 1998-9 PPS course. We have now set up a simple MUD at Birkbeck College for the exclusive use of students and tutors on Birkbeck's courses. Both the tutor and students may simultaneously display Web pages showing an assignment that forms the basis of the tutorial discussion.

Web communication can suffer from delays due to network congestion, particularly for traffic across the Atlantic. This is particularly important in countries with relatively poor network connections. Internet connections between Poland and the rest of the world became extremely slow during 1998 and part of 1999, due to network congestion in Warsaw. This problem has only recently been resolved (Sansom, 1999). In previous years we have taken up offers from other institutions to mirror our course materials in order to minimize these problems. These sites took nightly updates of the PPS course and in addition to providing faster Web communication, the mirrors give insurance against computer or network failures. However, since the teaching material for both courses is now password-protected, which makes mirroring technically harder, the mirrors have been removed. We hope to be able to reinstate at least the Polish mirror (at the Poznan Supercomputer Centre), which would make it easier for central European students to follow the courses.

\section{Evaluation}

At the end of 1998 we sent a questionnaire (Table 1), by email, to all 194 students who had taken the course during its first three years. The limitation of this means of communication is simply that many people change email addresses often, and at least forty questionnaires failed to reach their destinations. Therefore, the forty-five responses received represent a minimum 29 per cent response rate (45/154). Of the replies, nine $(12$ per cent of 75) came from students completing the course in 1996, 14 (26 per cent of 53) from those completing in 1997 and 21 (32 per cent of 66) from those completing in 1998. This improvement in response rate with students taking later courses probably just underlines the fact that students from earlier courses are more likely to have changed email addresses since completion.

A large majority of students agreed with positive statements about the course. Eightyseven per cent of the respondents (thirty-nine students) claimed that the course had 'broadly speaking' lived up to their expectations, and 80 per cent would recommend it to their colleagues. Many had already done so. Sixty-nine per cent agreed with the statement that the course was value for money. It is possible, however, that there was an element of self-selection, with those students who gained most from the course being most likely to 


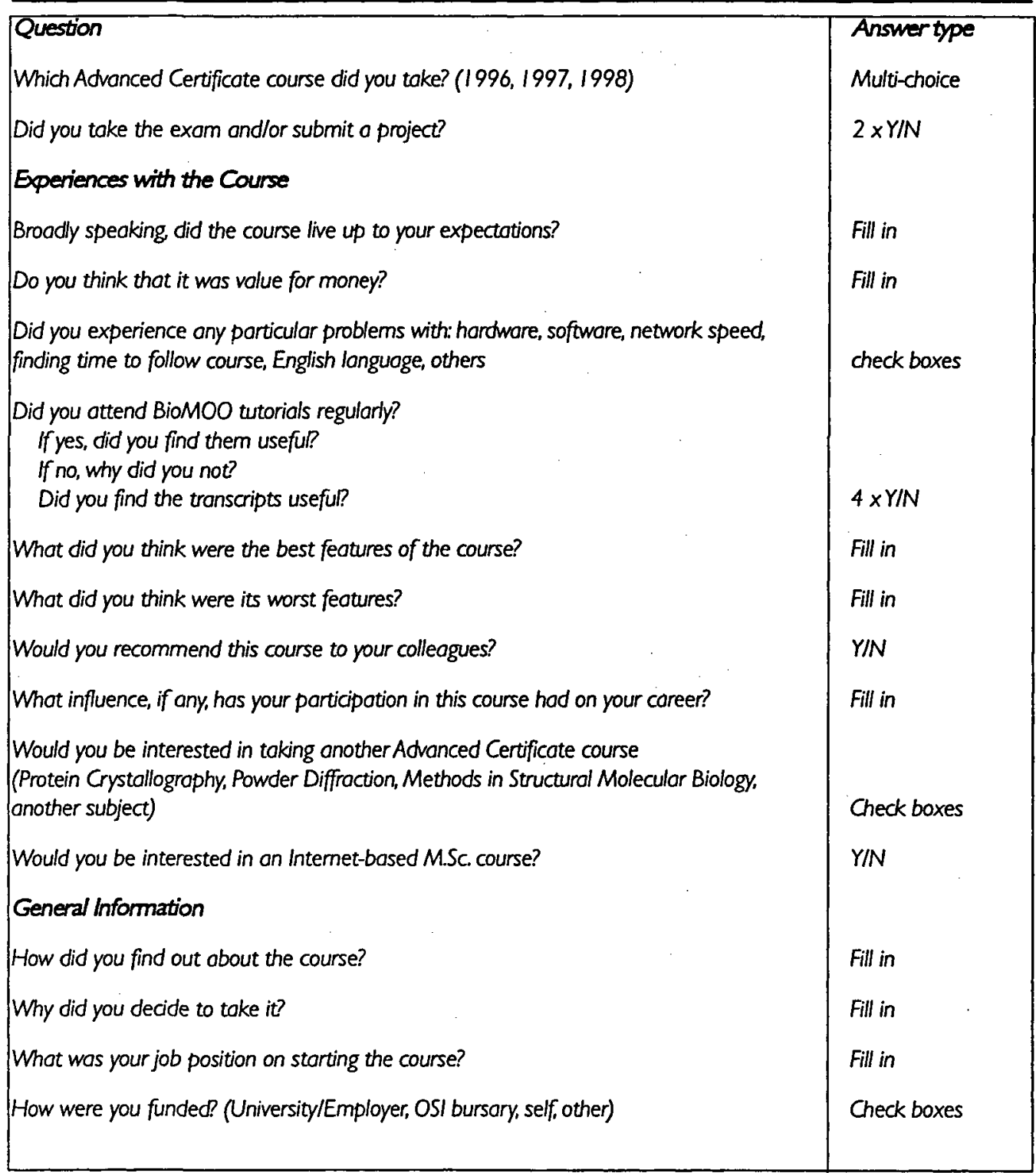

Table I: The questionnaire sent to former students of the Principles of Protein Structure course.

answer the questionnaire. When asked, without prompting, to name the best features of the course, the most common features mentioned were the convenient and flexible nature of this type of learning, the expertise of the tutors, and the Internet-based (non-specific) technology. The only specific section of the course material commended by more than one student was the unit on bioinformatics (in this context, the analysis of protein sequences).

There was much more consensus between respondents when asked to name the worst features of the course. No fewer than 27 per cent (twelve) of the students cited a lack of 


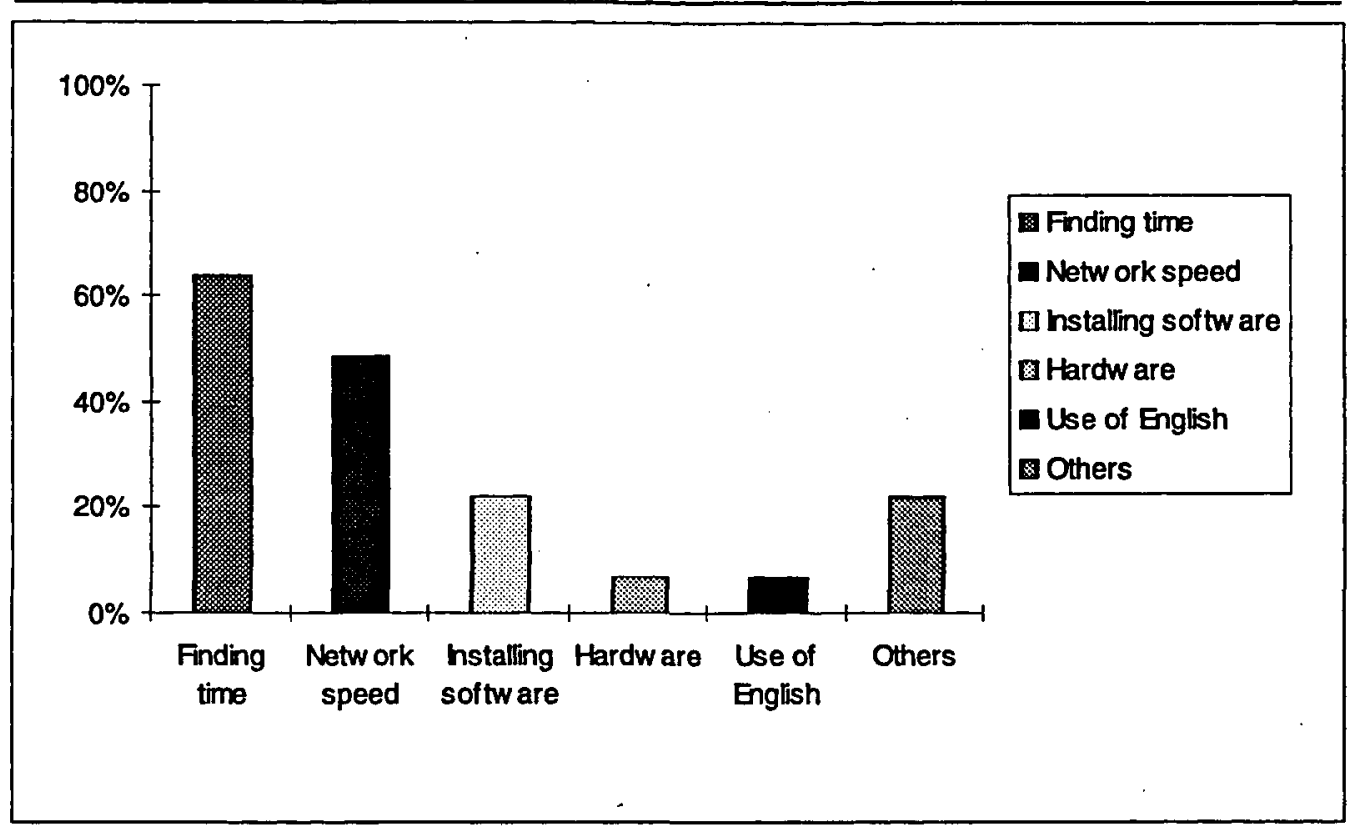

Figure 4: Percentages of questionnaire respondents reporting certain problems with following the PPS course.

supervision and feedback. This is a common feature of distance learning, but with the PPS course it was exacerbated by a lack of staff time. For many reasons most students found it difficult to attend tutorial sessions. Only 36 per cent of students reported having attended these 'regularly'. There are many reasons for this: difficulty in scheduling meetings (particularly for students based outside Europe and the Americas); other commitments; lack of confidence with written English at speed; and technical difficulties. A small minority of students, particularly those working in industry, has been unable to attend any due to 'firewalls'. Not all students have been happy to post scientific questions to the public mailing lists. The only other direct interaction between the students and their tutors occurs during the self-assessment periods. We have increased these from one to three a year, but it would be difficult to increase interaction with students beyond this without a significant further investment of staff time.

When prompted with a list of possible problems, 'finding time to follow the course' and 'network speed' were cited by more than half the students (Figure 4). A far higher proportion of students from central Europe than others found speed a problem; 73 per cent [11/15] of students supported by bursaries, compared with 40 per cent [12/30] of others mentioned that they had had problems with this. The mirror site in Poznan was removed when password protection of course material was introduced, and the Polish network infrastructure outside Warsaw was degraded in early 1998 due to competition between service providers (Sansom, 1999). This combination of circumstances caused Polish students taking the course in 1998-9 particular difficulties. 


\section{Future developments}

There are still some legal, technical and administrative problems that are hindering the wholesale acceptance of these teaching methods. One of the most serious of these concerns student assessment - when students and tutors never meet face to face, it is much harder to guard against plagiarism, or students collaborating on assignments - or even to ensure that the students registered on the course are those who take the examination. Issuing students with individual passwords, which is necessary for protein crystallography students, may be one way to minimize the risks. The qualification offered, the Advanced Certificate, is also much less well known than, for instance, the M.Sc., which is an international standard. Many potential students, particularly those located outside the UK, are unaware of the level of qualification it represents. Although Internet bandwidth is increasing rapidly, the equally rapid increase in 'traffic' means that communication delays are unlikely to improve much in the foreseeable future.

Recent hardware and software developments are already affecting the quality of our educational material and the ways in which the tutors, consultants and students can communicate. Applications written in Java can be used directly, over the Internet, on a variety of hardware platforms without needing installation. One example, CINEMA (Parry-Smith, Payne, Michie and Attwood, 1998) (a tool for aligning protein sequences), is currently used in the course. As network bandwidth increases, MOO-type programs will increasingly incorporate 'shared whiteboards' which will allow dispersed groups of people - for example, a tutorial group or a group of academic collaborators working on a paper to make changes to the same document in 'real time'. We are also investigating the simultaneous use of audio communication. Use of the higher bandwidth communication provided by ISDN or cable to provide audio and video may enable us to verify student identity locally.

We are gradually extending the range of subjects that we offer at Advanced Certificate level using this technology. We have just introduced a third Advanced Certificate, in Powder Diffraction, and we hope to introduce a fourth, Techniques in Structural Molecular Biology, during 2001. We are also intending to introduce an Internet-based part-time modular M.Sc. course, which will be built up from the Advanced Certificates. Most students would take the PPS course in the first year of M.Sc. study, followed by one of the more advanced courses. Students may also be required to submit a project.

\section{Acknowledgements}

The authors would like to thank Dr Huub Driessen for useful discussions.

\section{References}

Branden, C. and Tooze, J. (1999), Introduction to Protein Structure, Second Edition, New York: Garland Publishing, Inc.

Farrell, G. M. (1999), The Development of Virtual Education: A Global Perspective, The Commonwealth of Learning, http://www.col.org/virtualedlindex.htm. 
King, T. (1998), 'Towards a strategy for the re-use of legacy teaching materials in Webbased courses', Active Learning, 9, 9-14.

MDLI, Inc. (1999), Chime v.2.0.2, http://www.mdli.com/support/chimeldefault.html, or http://www.umass.edulmicrobio/chimel.

Parry-Smith, D. J., Payne, A. W. R, Michie, A. D. and Attwood, T. K. (1998), 'CINEMA a novel colour interactive editor for multiple alignments', Gene, 211(2), 45-56.

Sansom, C. E. (1997), 'BioMOO: teaching biology in virtual reality', Life Sciences Educational Computing, 8(3), 20-2.

Sansom, C. E., Walshaw, J. and Moss, D. S. (1997), 'Principles of protein structure', The Biochemist, October 1997, 14-17.

Sansom, C. E. (1999), 'European biotechnology turns east', Nature Biotechnology, 17, 437-9.

Sayle, R. (1996), RasMol v. 2.6. (Molecular graphics viewer),

http://www.umass.edulmicrobio/rasmoll.

Towell, J. F, and Towell, E. R. (1997), 'Presence in text-based networked virtual environments or "MUDS", Presence, 6, 590-5. 